\title{
RESVERATROL AND CURCUMIN AS PROTECTIVE AGENTS IN AN EXPERIMENTAL RAT MODEL OF INTESTINAL ISCHEMIA AND REPERFUSION
}

\begin{tabular}{|r|l|}
\hline Journal: & Canadian Journal of Physiology and Pharmacology \\
\hline Manuscript ID & cjpp-2016-0085.R1 \\
\hline Manuscript Type: & Article \\
\hline Date Submitted by the Author: & $19-$ Apr-2016 \\
\hline Complete List of Authors: & $\begin{array}{l}\text { Cucolas, Cristina; "Iuliu Haţieganu" University of Medicine and Pharmacy, } \\
\text { Cluj-Napoca, Romania , Department of Physiology } \\
\text { Daneasa, Alexandra; "Iuliu Haţieganu" University of Medicine and } \\
\text { Pharmacy } \\
\text { Olteanu, Diana; "Iuliu Haţieganu" University of Medicine and Pharmacy, } \\
\text { Physiology } \\
\text { Decea, Nicoleta; University of Medicine and Pharmacy, Physiology } \\
\text { Moldovan, Remus; University of Medicine and Pharmacy, Physiology } \\
\text { Tabaran, Flaviu; Universitatea de Stiinte Agricole si Medicina Veterinara din } \\
\text { Cluj-Napoca } \\
\text { Filip, Gabriela; University of Medicine and Pharmacy, Physiology }\end{array}$ \\
\hline Keyword: & \begin{tabular}{l} 
resveratrol, curcumin, oxidative stress, inflammation, intestinal ischemia \\
\hline
\end{tabular} \\
\hline
\end{tabular}




\title{
RESVERATROL AND CURCUMIN AS PROTECTIVE AGENTS IN AN EXPERIMENTAL RAT MODEL OF INTESTINAL ISCHEMIA AND REPERFUSION
}

\author{
Cristina Cucolas ${ }^{1}$, Alexandra loana Daneasa $^{1}$, Diana Olteanu $^{1}$, Nicoleta Decea ${ }^{1}$,
}

Remus Moldovan ${ }^{1}$, Flaviu Tabaran ${ }^{2}$, Gabriela Adriana Filip ${ }^{1 \S}$

${ }^{1}$ Department of Physiology, "Iuliu Haţieganu” University of Medicine and Pharmacy, ClujNapoca, Romania

${ }^{2}$ Department of Pathology, University of Agricultural Sciences and Veterinary Medicine, 400372, Cluj-Napoca, Romania

$\S$ Corresponding author

Gabriela Adriana Filip

Address: Clinicilor Street, no 1, Cluj-Napoca, Romania

Phone: 0040-745-268704 Fax: 0040-264-598575

e-mail: gabriela.filip@umfcluj.ro; adrianafilip33@yahoo.com 


\section{Abstract}

The aim of this study was to evaluate the protective effects of resveratrol and curcumin in an experimental rat model of intestinal ischemia-reperfusion (I/R). 48 adult Wistar rats were used: 12 animals undergoing the sham surgery and 36 animals undergoing laparotomy, with 15 minutes of mesentric artery clamping. The animals from the latter groups $(n=12)$ were pre-treated, for a week, with vehicle (CTR), resveratrol (RES) and curcumin (CUR). After one and 6 hours of reperfusion respectively, cyclooxigenase (COX)-2, mucin-1, E-cadherin, nuclear factor (NK)-kB expressions and tumor necrosis factor-related apoptosis-inducing ligand (TRAIL) were assessed in the small intestine. Oxidative stress markers were determined in tissue homogenate and serum and histopathological analysis was performed. Pretreatment with RES decreased the expression of COX-2 and NF-kB at both intervals and increased E-cadherine $(p<0.05)$ and mucin-1 production, after one hour. CUR had a beneficial effect on COX-2, NF-kB and E-cadherine expressions, both after one and 6 hours $(p<0.0001)$. The two compounds increased TRAIL levels and had a protective effect on oxidative stress and histopatological lesions, both after one and 6 hours. Our results suggested that RES and CUR had beneficial effects in intestinal ischemia and may represent a promising option for complementary treatment of this pathological condition.

Key words: resveratrol, curcumin, oxidative stress, inflammation, intestinal ischemia 


\section{Introduction}

Acute mesenteric ischemia, due to reduced splanchnic circulation followed by early revascularization, is the underlying mechanism that describes the phenomenon of intestinal ischemia-reperfusion $(I / R)$. Intestinal $I / R$ is not only a clinical problem in the perioperative settings such as vascular surgery, trauma, intestinal and liver transplantation (Kim et al. 2012) but also during stricture, gangrene, fulminant universal colitis; it always claims a challenging diagnosis and management (Wang et al. 2012).

Intestinal I/R is associated with a high mortality and morbidity rate because it affects remote organs such as the lungs, liver and kidneys and predisposes to systemic inflammation and multi-organ failure (Fan et al. 2014). In intestinal I/R physiopathology, two different stages can be noticed: the ischemic stage in which ATP decreases, membrane potentials and calcium ion distribution are altered, acidosis and activation of cytotoxic enzymes appear; and the reperfusion stage characterized by increased production of oxygen reactive species and epithelial barrier dysfunction (Katada et al. 2015). Latest studies have shown that disruption of the epithelial-mucosal barrier gives access to the digestive enzymes into de intestinal wall, consequently triggering its autodigestion (Chang et al. 2012). Mucine from mucus and epithelial membrane proteins such as E-cadherine seems to be degraded in the early periods and leads to systemic inflammation and hyperexpression of COX-2 (Wang et al. 2012). In case of loss of tissue integrity, an important role in the initiation and progression of inflammation is played by neutrophils. A recent study showed that up-regulation of the tumor necrosis factor-related apoptosis-inducing ligand (TRAIL) in nonmalignant cells promotes neutrophil apoptosis and limits inflammation (Leitch et al. 2011). 
Although it is known that the duration of the ischemic period directly influences the gravity of the lesions, several cytoprotective mediators such as nitric oxide, carbon monoxide, prostaglandins and isoflurane have been studied as a therapeutic target for intestinal I/R injury (Katada et al. 2015; Kim et al. 2012).

Resveratrol $\left(3,5,4^{\prime}\right.$-trihydroxystilbene) is a natural polyphenol belonging to the phytoalexin family which has demonstrated protective effect due to antioxidant, antiinflammatory, antiaging, wound healing and pro/anti-apoptotic properties by targeting different molecules such as endothelial nitric oxide synthase (eNOS), estrogen receptors and nuclear factor-kappa B (NF-kB) (Su et al. 2013). Resveratrol has also been suggested to prevent and treat ischemia and reperfusion injury due to its antioxidative activities (Liu et al. 2015). Curcumin (1,7-bis(4-hydroxy-3-methoxy-phenyl)-1,6-heptadiene-3, 5 dione) is a yellow-orange dye extracted from the Indian spice turmeric. It has been reported to possess pleiotropic effects as antioxidant, anti-carcinogenic, antibacterial, antiviral, antiinflammatory, anti-proliferative, and pro-apoptotic (Katada et al. 2015). Curcumin administration has been shown to ameliorate $\mathrm{I} / \mathrm{R}$ injury in the rat intestine, liver, kidney, and nervous tissue but the mechanisms are not fully understood (Fan et al. 2014; Saleh and Sherif 2014).

Based on these data, the aim of our study is to compare both local and systemic protective effects of RES and CUR pretreatment using biochemical and histopathological investigations. In order to do that, oxidative stress markers, NF-kB/pNF-kB, COX-2, mucin-1 and E-cadherine expressions and TRAIL levels in intestinal samples were evaluated.

\section{Methods}


Trichloroacetic acid, resveratrol, curcumin, Bradford reagent, o-phtalaldehyde and rabbit antibodies for mucin-1 and E-cadherin were purchased from Sigma-Aldrich Chemicals GmbH (Germany), 2-thiobarbituric acid from Merck KgA Darmastadt (Germany) and absolute ethanol and n-butanol were provided by Chimopar (Bucharest). ELISA Immunoassay kit for evaluation of TRAIL levels was obtained from R\&D Systems (Inc. Minneapolis, USA) and antibodies for COX-2, pNFkB p65 (Ser 311), NFkB p65, secondary antibody mouse anti-goat and glyceraldehyde 3-phosphate dehydrogenase (GAPDH) were purchased from Santa Cruz Biotechnology (Heidelberg, Germany). Supersignal West Femto Chemiluminiscent substrate was from Thermo Fisher Scientific (Rockford IL, USA).

\subsection{Experimental design}

Forty-eight adult male Wistar rats $(225 \pm 25 \mathrm{~g})$ were used. The animals were obtained from the Animal Department of "Iuliu Hațieganu" University of Medicine and Pharmacy, ClujNapoca, Romania. For acclimatization, they were housed in the Physiology Department's Biobase a week before under controlled temperature of $22^{\circ}-24^{\circ} \mathrm{C}, 12 \mathrm{~h}$ light - $12 \mathrm{~h}$ dark cycle, free access to pellet food and water. All experiments were performed according to the approved animal care protocols of the Ethics Committee on Animal Welfare of the "Iuliu Hațieganu" University (as a part of project no 1493/6/28.01.2014) in accordance with European Convention for the Protection of Vertebrate Animals used for Experimental and other Scientific Purposes, Council of Europe no. 123, Strasbourg 1985.

The rats were divided into four groups ( $n=12)$ as follows: sham group (SHAM), control group (CTRL) pretreated orally with normal saline, resveratrol group (RES) pre-treated orally with $15 \mathrm{mg} / \mathrm{kg}$ body weight (b.w.) resveratrol and curcumin group (CUR) pretreated orally with $15 \mathrm{mg} / \mathrm{kg}$ curcumin, both in saline vehicle. The treatment was performed for one week and then all 48 animals underwent surgery as follows: simple xifo-pubian laparotomy with 
isolation of the emergence of the superior mesenteric artery (SMA) followed by laparorrhaphy for the sham group and a xifo-pubian laparotomy with isolation and 15 minutes clamping of the SMA with a vascular clamp followed by laparorrhaphy for the other 3 groups. Each group was further divided into two groups $(n=6)$ depending on the time of reperfusion, one and 6 hours respectively. After this time, the animals were euthanized; small intestine and blood samples were harvested for biochemical and histopathological analyses. Markers of oxidative stress in intestine homogenates were evaluated and serum and COX-2, mucin-1, E-cadherin, NF-kB, pNF-kB expressions were quantified by western blot and TRAIL by ELISA in tissue samples. All the invasive procedures were performed under combined anesthesia with ( $90 \mathrm{mg} \mathrm{kg}^{-1}$ ketamine, $10 \mathrm{mg} \mathrm{kg}^{-1}$ xylazine).

\subsection{Oxidative stress assay}

For oxidative stress assessment, malondyaldehide (MDA) and glutahione reduced/oxidized (GSH/GSSG) ratio were performed in small intestinal tissue and serum. The intestine samples were prepared as previously described (Olteanu et al. 2012). The protein content was measured with Bradford method (Noble and Bailey 2009). Samples from each animal were stored in aliquots at $-80^{\circ} \mathrm{C}$ until assayed.

\subsection{Western Blot analysis}

Small intestine samples ( $20 \mu \mathrm{g}$ of protein/lane) were subjected to SDS-PAGE (12\% polyacrylamide) as previously described (Olteanu et al. 2012). Blots were blocked for 1 hour at room temperature and incubated with the primary antibody (1:1000) against COX-2, mucin-1, E-cadherin,NF-kB and pNF-kB. Subsequently, the membranes were incubated with corresponding secondary peroxidase-coupled antibody (1:1500). GAPDH served as 
endogenous control. Proteins were detected using Supersignal West Femto Chemiluminiscent substrate (Thermo Fisher Scientific, Rockford IL, USA) and analyzed using Phoretix array (free trial version).

\subsection{ELISA evaluation of TRAIL}

The TRAIL immunoassay kit was used according to manufacturer's instructions. The results were expressed in $\mathrm{pg} / \mathrm{mg}$ protein.

\subsection{Histology analysis of intestinal samples}

After necropsy, the intestinal content of the jejunum (full length) was removed by luminal perfusion with saline and fixed in $10 \%$ neutral-buffered formalin (NBF). After $48 \mathrm{~h}$ the samples were submerged to dehydration in ethanol, clarification in xylene and finally embedded in paraffin-wax following the routine technique. Multiple tissue sections were cut from each paraffin block, at $4 \mu \mathrm{m}$ thickness, with a rotary microtome (Leica RM RT 2125) and finally stained with hematoxylin - eosin (H\&E). Histological slides were examined under an Olympus BX41 microscope and micro-images were obtained by an Olympus UC30 camera (Dăneasă et al. 2014).

\subsection{Statistical analysis}

Data obtained were analyzed with GraphPad Prism Software, version 5.0 (San Diego, CA) with Bonferroni's multiple comparison test as posttest. All values in text and figures were expressed as mean \pm standard deviation, with a limit of statistical significance of $p<0.05$.

\section{Results}

\subsection{The effects of RES and CUR administration on oxidant/antioxidant status}

The oxidative stress induced by intestinal ischemia was evaluated by determination 
of MDA levels, both in intestinal and blood samples. A rather normal tendency was noticed in the CTR groups where the MDA levels enhanced as compared to SHAM groups $(p<0.05)$. A decrease of MDA levels were noticed in the pretreated CUR group $(p<0.05)$ after one hour of reperfusion in the intestine samples as compared to controls (Figure 1a). An increase of the antioxidant capacity was observed, both after one and after six hours of reperfusion, in the intestinal samples pretreated with CUR $(p<0.05)$ as compared to controls (Figure $1 \mathrm{~b}$ ). MDA levels diminished in serum after six hours of reperfusion, in the RES group $(p<0.01)$, in parallel with improving of GSH/GSSG ratio $(p<0.01)$ (Figure $1 c)$. The pretreatment with CUR diminished the GSH/GSSG ratio in serum after 1 hour of reperfusion without influencing the MDA formation ( $p>0.05$ ) (Figure $2 d$ ).

3.2. The effects of RES and CUR administration on COX-2, NF-kB, pNF-kB, mucin-1, Ecadherin and TRAIL expressions

In order to evaluate inflammation in the small intestine under $I / R$ and the effects of RES and CUR pretreatments, the expressions of COX-2, NF-kB, pNF-kB , mucin-1, E-cadherin and TRAIL levels were assessed (Figure 3g).

The expression of COX-2 in intestinal homogenates showed a significant decrease in RES group after one hour $(p<0.01)$ as compared to controls (Figure $3 a)$. The values diminished significantly after six hours in both treated groups ( $p<0.0001)$ (Figure $3 b)$.

NF-kB levels decreased statistically significantly $(p<0.0001)$ in the RES pretreated group, one hour and six hours after reperfusion. CUR had the same effect only after six hours $(p<0.0001)$ (Figure $3 c$ and $3 d)$. RES and CUR administration increased statistically significantly the activation of NF-kB $(p<0.0001)$, both after one and six hours after reperfusion, as compared to controls (Figure $3 e, 3 f)$. 
E-cadherin expression diminished in the group that underwent the ischemia reperfusion and treated with vehicle, compared to the sham group and increased significantly in group pretreated with RES, after one hour of reperfusion $(p<0.01)$ (Figure 4a). CUR administration before $\mathrm{I} / \mathrm{R}$ improved the expression of this protein, both after one $(p<0.0001)$ and six hours of reperfusion $(p<0.001)$ (Figure $4 b)$. Only in the RES group the intestinal mucin-1 expression increased after one hour $(p<0.001)$ of reperfusion as compared to controls (Figure 4c, 4b).

TRAIL levels in intestine samples showed that the molecule was up-regulated in the RES group after one hour $(p<0.0001)$ and after 6 hours $(p<0.5)$, but also in both CUR groups $(p<0.001)$ as compared to controls (Figure $5 a, 5 b)$.

\subsection{The effects of RES and CUR administration on histopathological sections}

In order to evaluate the morphological alterations induced by $l / R$, the intestine samples were harvested from all the animals included in the study. The samples were compared to the correspondent control group, depending on the time of reperfusion, one and six hours respectively (Figure 6). Normal villi with intact epithelial cells were observed in the sham groups. Superficial erosions and mild congestion or light inflammatory infiltrate of the lamina propria were noticed in all the experimental groups that underwent ischemia, after one hour of reperfusion. A multifocal or diffuse distribution of the above mentioned lesions could be seen strictly limited to the intestinal mucosa. In addition, the necrosis of the intestinal villi was observed although with a high degree of variability between groups. Thus, in the control group, necrosis affected approximately half of the villi length while in the RES respectively CUR pretreated groups; necrosis was noticed strictly in the superficial areas (Figure 6, images B, C and D). Six hour after ischemia, in the control group, transmural 
necrosis, multifocal inflammation followed by subsequent massive edema of the tissue was noticed. In both pretreated groups, the necrosis had a superficial, multifocal aspect and affects only the intestinal epithelium. Moreover, superficial necrosis, moderate congestion and inflammatory infiltrate of the jejunal mucosa were present in the protected groups. In order to differentiate the levels of tissue damage we used the scoring system published by Chiu at al (Chiu et al. 1970). Thus, both SHAM groups were graded 0 (normal villi), grade 4 (denuded villi) for the CTR group, after 1 hour of reperfusion, respectively grade 5 (disintegration of the lamina propria) for CTR group after 6 hours of reperfusion. Regarding the pretreated groups, both RES groups were grade 4 (denuded villi with lamina propria, dilated capillaries exposed and increased cellularity), while both CUR groups were graded 3 (marked epithelial lifting down the sides and a few denuded villi tips).

\section{Discussion}

The present study investigated the protective effects of two natural compounds, resveratrol and curcumin, on an experimental rat model of intestinal I/R. The effects were quantified by oxidative stress parameters in serum and small intestine samples and markers of inflammation, both after one and after six hours of reperfusion. For testing we chose two compounds known for their beneficial effects, low toxicity and affordability.

Intestinal I/R represents a severe event which often follows mesenteric embolism, intestinal disease sepsis, transplantation or hemorrhagic shock and is thought to be an inducer of multiple organ dysfunction syndrome (MODS) (Chiu et al. 1970). The gastrointestinal tract, highly sensitive to $\mathrm{I} / \mathrm{R}$, could trigger the release of oxidants and tissue damaging factors, which cause intestinal edema, microvascular permeability changes, vasoregulation impairment, mucosal barrier dysfunctions and amplify inflammatory cellular 
infiltration (Liu et al. 2015). The epithelial-mucosal barrier is fortified by various cell adhesion molecules such as E-cadherin and a mucus layer containing different types of mucin, proteins altered by $\mathrm{I} / \mathrm{R}$ injury as shown by Turner (Turner 2009). Our results demonstrated that RES, administered one week before the ischemic event, restored the levels of mucin-1 after one hour of reperfusion and increased significantly the expression of E-cadherin $(p<0.01)$. CUR maintained the high levels of E-cadherin, comparable to RES, both after one and 6 hours. These results suggested that while RES showed a beneficial effect on mucin-1 and Ecadherin, CUR only influenced the E-cadherin levels but more significantly than RES and for a longer period of time.

Several studies have shown that both CUR and RES have tissue protective and wound healing properties, in a wide range of pathologies, including intestinal I/R (Fan et al. 2014; Liu et al. 2015; Saleh and Sherif 2014). Our study showed that CUR administration diminished the MDA levels and increased the GSH/GSSG ratio in intestine samples. The protective role of CUR on oxidative stress was early and maintained after six hours, thus RES had a slightly delayed effect, being noticed in the blood samples only after six hours. The histopathological aspects were consistent with those of oxidative stress. Thus, both SHAM groups were free of alterations. Severe damages were noticed in the CTR groups, with a more dramatic aspect after six hours of reperfusion. Both protected groups showed lower levels of inflammation, necrosis and loss of tissue integrity, as compared to controls. Moreover, in the CUR group the architecture of the cells was slightly better than in the RES group, both after one and six hours of reperfusion.

Our results are in agreement with other findings. Thus, pterostilbene, a naturally derived compound similar to resveratrol, decreased oxidative stress induced by intestinal I/R and reduced the upregulation of COX-2 and NF-kB (Sun et al. 2014). COX-2 expression, as 
marker of inflammation evaluated by western blot analysis, increased significant in the control groups, mainly after 6 hours. RES treatment maintained very low levels of COX-2, similar to the sham groups, both after one hour and after 6 hours while the CUR, although also suppressed the inflammation, only had significant results after 6 hours. These results could be explained by antimicrobial effects of RES because it not only protects the integrity of the tissue, but also, in case of disruption of the barrier, it might also act as an antimicrobial agent (Taylor et al. 2014). TRAIL, a relatively recent discovered molecule, a member of the TNF family has two opposite effects. On the one hand it induces apoptosis by binding to the DR4 and DR5 receptors and on the other it can have an anti-apoptotic NF-kB dependent effect by binding of the decoy receptors, DcR1 and DcR2 (Kichev et al. 2014; Vries et al. 2006). It is a known fact that TRAIL apoptosis inducer effect is only related to cancer cells, it's role regarding normal cells being a subject that still needs to be studied.

A recent study demonstrated that during ischemia, the levels of E-cadherin diminished. E-cadherin is known as a surviving signaling molecule sent from the extracellular matrix to the epithelial cells and can induce a TRAIL mediated apoptosis. The study also mentioned a rapid reparative response of the epithelial continuity due to a tight junction and endogenous cytosolic proteins (Derikx et al. 2008). In our study, although mucin-1 was elevated only in the RES group after one hour, E-cadherin levels were significant higher after this pretreatment suggesting the wound healing effect of our compounds.

Several studies have demonstrated the pro-apoptotic, TRAIL up-regulating, effects of CUR and RES (Park et al. 2013; Tameda et al. 2014). Thus, the two compounds increased the expression of TRAIL, both after one hour and after 6 hours, for CUR and only after one hour for RES. We hypothesize that the up-regulating effect of TRAIL might not only lead to the shedding of mature apical epithelial layer affected by ischemia but also work as a protective 
mechanism through the DcR1 and DcR2 receptors, on the NF-kB dependent pathway. Besides wound healing properties, the damage dependent effect of TRAIL could make RES and CUR two ideal rescue elements of the mucosal/epithelial barrier in intestinal I/R. This idea hasn't, to our knowledge, been suggested before and further studies should certainly be done in order to better analyze the mechanisms.

The I/R syndrome has been thoroughly studied on a variety of organs and a rather large number of natural antioxidants have been tested. Thus, huperzine A, an alkaloid found in Huperzia serrata and magnolol, a lignin compound from Magnolia officinalis, have been shown to have beneficial effects on brain I/R in rats (Venkatesan and Kim 2015). Moreover, quercetin, a natural flavonoid, had protective effects in I/R of the retina (Arikan et al. 2015) due to its anti apoptotic effect. Quercetin also had antioxidant activity and led to radicalscavenging, xanthine oxidase inhibition and chain breaking effects in I/R of intestine (Itagaki et al. 2010).

\section{Conclusions}

Our results confirm that RES and especially CUR are two promising ways of protection against intestinal $I / R$, not only on a systemic level due to their antioxidant capacity but also due to the important benefits on mucosal/epithelial barrier. Moreover, the low toxicity and the rather affordable prices make them encouraging treatment methods, suitable for clinical usage but especially as preventive therapy for at risk populations.

\section{Authors' contributions}

CC designed the experiments and wrote the paper. AD performed the statistical analysis for oxidative stress parameters and drafted the manuscript. RM designed and implemented the 
research study. ND carried out and analyzed the biochemical parameters. TF performed the histopathological analysis and DO the western blot investigations. GAF did the statistical analysis for oxidative stress parameters, wrote the paper and gave the final approval. All authors read and approved the manuscript.

\section{Acknowledgements}

This study was financed by the University of Medicine and Pharmacy "Iuliu Hațieganu" ClujNapoca, Romania, internal grant no. 1493/6/28.01.2014. The funders had no role in study design, data collection and analysis, decision to publish, or preparation of the manuscript.

\section{Declaration of interest}

The authors have no competing interest.

\section{References}

Arikan, S., Ersan, I., Karaca. T., Kara, S., Gencer, B., Karaboga, I., et al. 2015. Quercetin protects the retina by reducing apoptosis due to ischemia-reperfusion injury in a rat model. Arq. Bras. Oftalmol. 78(2):100-4.

Chang, M., Kistler, E.B., and Schmid-Schönbein, G.W. 2012. Disruption of the Mucosal Barrier During Gut Ischemia Allows Entry of Digestive Enzymes Into the Intestinal Wall. Shock, 37(3):297-305.

Chiu, C.J., AcArdle, A.H., Brown, R., Scott. H.J., and Gurd, F.N. 1970. Intestinal Mucosal Lesion in Low-Flow States I. A Morphological, Hemodynamic, and Metabolic Reappraisal. 
Arch. Surg. 101(4):478-483.

Dăneasă, .A, Cucolaș, C., Furcea, M., Bolfa, P., Dudea, S., Olteanu, D., et al. 2014. Spironolactone and dimethylsulfoxide effect on glucose metabolism and oxidative stress markers in polycystic ovarian syndrome rat model. Exp. Clin. Endocrinol. Diabetes, $122(3): 154-62$.

Derikx, J.P.M., Matthijsen, R ., de Bruïne, A.P., van Bijnen, A., Heineman, E., van Dam, R.M., et al. 2008. Rapid reversal of human intestinal ischemia-reperfusion induced damage by shedding of injured enterocytes and reepithelialisation. PLoS One, 3(10):e3428.

Fan, Z., Jing, H., Yao, J., Li, Y., Hu, X., Shao, H., et al. 2014. The Protective Effects of Curcumin on Experimental Acute Liver Lesion Induced by Intestinal Ischemia-Reperfusion through Inhibiting the Pathway of NF- $\kappa$ B in a Rat Model. Oxid. Med. Cell Longev. 2014: 1-8.

Itagaki, S., Oikawa, S., Ogura, J., Kobayashi, M., Hirano, T., and Iseki, K. 2010. Protective effects of quercetin-3-rhamnoglucoside (rutin) on ischemia-reperfusion injury in rat small intestine. Food Chem. 118(2):426-9.

Katada, K., Takagi, T., Uchiyama, K., and Naito, Y. 2015. Therapeutic roles of carbon monoxide in intestinal ischemia-reperfusion injury. J. Gastroenterol. Hepatol. 30:46-52.

Kichev, A., Rousset, C.I., Baburamani, A.,, Levison, S.W., Wood, T.L., Gressens, P., et al. 2014. Tumor necrosis factor-related apoptosis-inducing ligand (TRAIL) signaling and cell death in 
the immature central nervous system after hypoxia-ischemia and inflammation. J. Biol. Chem. 289(13):9430-9.

Kim, M., Park, S.W., Kim, M., D'Argati, V.D., and Lee, H.T. 2012. Isoflurane postconditioning protects against intestinal ischemia-reperfusion injury and multi-organ dysfunction via transforming growth factor-beta1 generation. Ann. Surg. 255(3):492-503.

Leitch, E., Lucas, C.D., and Rossi, G. 2011. Editorial: Neutrophil apoptosis: hot on the TRAIL of inflammatory resolution. J. Leukoc. Biol. 90(5):841-3.

Liu, F.C., Tsai, H.I., and Yu, H.P. 2015. Organ-Protective Effects of Red Wine Extract, Resveratrol, in Oxidative Stress-Mediated Reperfusion Injury.(Table 1) Oxid. Med. Cell. Longev. 2015: Article ID 568634. http://dx.doi.org/10.1155/2015/568634

Noble, J.E., Bailey, M.J. 2009. Chapter 8 Quantitation of Protein. Methods Enzymol. 73-95.

Olteanu, D., Nagy, A., Dudea, M., Filip, A., Muresan, A., Catoi .C, et al. 2012. Hepatic and systemic effects of rosuvastatin on an experimental model of bile duct ligation in rats. J. Physiol. Pharmacol. 63(7):483-96.

Park, S., Cho, D.H., Andera, L., Suh, N., and Kim, I. 2013. Curcumin enhances TRAIL-induced apoptosis of breast cancer cells by regulating apoptosis-related proteins. Mol. Cell. Biochem. $383(1-2): 39-48$.

Saleh, D., and Sherif, R. 2014. The Protective Effect of Curcumin versus Sodium Nitroprusside on Intestinal Ischemia/Reperfusion Injury. J. Interdiscip. Histopathol. 2(74):1. 
Su, D., Cheng, Y., Liu, M., Liu, D., Cui, H., Zhang, B., et al. 2013. Comparision of Piceid and Resveratrol in Antioxidation and Antiproliferation Activities In Vitro. PLoS One, 8(1).

Sun, Y., Xu, Y., and Wang, G.N. 2015. Pterostilbene Prevents Intestinal Ischemia Reperfusion Injury in Wistar Rats via Modulation of Antioxidant Defense and Inflammation. Tropical Journal of Pharmaceutical Research, 14(8): 1383-91.

Tameda, M., Sugimoto, K., Shiraki, K., Inagaki, Y., Ogura, S., Kasai, C., et al. 2014. Resveratrol sensitizes HepG2 cells to TRAIL-induced apoptosis. Anticancer Drugs, 25(9):1028-34.

Taylor, E.J.M., Yu, Y., Champer, J., and Kim, J. 2014. Resveratrol Demonstrates Antimicrobial Effects Against Propionibacterium acnes In Vitro. Dermatol. Ther. (Heidelb). 4(2):249-57.

Turner, J.R.. 2009. Intestinal mucosal barrier function in health and disease. Nat Rev Immunol. 9(11): 799-809.

Venkatesan, R., Ji, E., and Kim, S.Y. 2015. Phytochemicals That Regulate Neurodegenerative Disease by Targeting Neurotrophins: A Comprehensive Review. Biomed. Res. Int. 2015:1-22.

Vries, E De., Gietema, J., and Jong, S De. 2006. Tumor Necrosis Factor-Related ApoptosisInducing Ligand Pathway and Its Therapeutic Implications. Clin. Cancer Res. 12(8):2390-3.

Wang, F., Li, Q., Wang, C., Tang, C., and Li, J. 2012. Dynamic Alteration of the Colonic Microbiota in Intestinal Ischemia-Reperfusion Injury. PLoS One, 7(7):e42027. 


\section{Figure legends:}

Figure 1- Oxidative stress parameters from serum in I/R after one week of pretreatment with RES and CUR. (a) Malondialdehyde levels in serum after one hour of reperfusion; (b) GSH/GSSG ratio after one hour of reperfusion; (c) MDA production after six hours of reperfusion; (d) GSH/GSSG ratio after six hours of reperfusion. The statistical significance betwen compared groups was evaluated with one-way ANOVA followed by Bonferroni posttest, ${ }^{*} p<0,5, * * p<0,01, * * * p<0,001, * * * * p<0,0001$.

Figure 2 - Oxidative stress parameters in intestine samples in $1 / R$ after one week of pretreatment with RES and CUR. (a) Malondialdehyde levels after one hour of reperfusion; (b) GSH/GSSG ratio after one hour of reperfusion; (c) MDA formation after six hours of reperfusion; (d) GSH/GSSG ratio after six hours of reperfusion. The statistical significance betwen compared groups was evaluated with one-way ANOVA followed by Bonferroni posttest, $* p<0,5, * * p<0,01, * * * p<0,001, * * * * p<0,0001$

Figure 3 - Western Blot analysis of inflammatory markes in intestine samples in I/R after one week of pretreatment with RES and CUR. $(a, b)$ COX-2 expressions after one hour and six hours of reperfusion. Image analysis of Western blot bands' intensity was performed through densitometry and the data, normalized to GAPDH, are shown as graphs in upper panels. (c, d) NF-kB expressions after one hour and six hours of reperfusion; (e, f) pNF-kB levels after one hour and six hours of reperfusion. (g) Representative images of immunoblotting for COX2, NF-kB, pNF-kB and GAPDH in intestine homogenates are shown in lower panel. The statistical significance betwen compared groups was evaluated with one-way ANOVA 
followed by Bonferroni post-test, ${ }^{*} p<0,5, * * p<0,01, * * * p<0,001, * * * * p<0,0001$.

Figure 4 - E-cadherin and mucine-1 expressions in intestine samples in I/R after one week of pretreatment with RES and CUR. (a, b) E-cadherin expressions after one and six hour of reperfusion. Image analysis of Western blot bands' intensity was performed through densitometry and the data, normalized to GAPDH, are shown as graphs in upper panels.

(c, d) Mucin-1 levels after one hour and six hours of reperfusion. (e) Representative images of immunoblotting for E-cadherin, mucin-1 and GAPDH in intestine samples are shown in lower panel. The statistical significance betwen compared groups was evaluated with oneway ANOVA followed by Bonferroni post-test, ${ }^{*} p<0,5, * * p<0,01, * * * p<0,001, * * * * p<0,0001$.

Figure 5 - TRAIL level in intestine samples in intestinal I/R after one week of pretreatment with RES and CUR. (a, b) TRAIL levels after one hour and six hours of reperfusion. The statistical significance betwen compared groups was evaluated with one-way ANOVA followed by Bonferroni post-test, ${ }^{*} p<0,5, * * p<0,01, * * * p<0,001, * * * * p<0,0001$.

Figure 6 - Histopathological images in intestinal I/R after one week of pretreatment with RES and CUR. (A) - normal intestinal morphology in the SHAM group one hour after surgery; (B) multifocal mucosal erosions and superficial necrosis (arrow) of the jejunum one hour following ischemia, severe congestion and mild inflammatory infiltrate (asterisk) of the lamina propria; (C) - focal mucosal erosions (arrows), congestion and mild inflammation (asterisk) in the lamina propria one hour following ischemia in RES pretreated group; (D) diffuse intestinal erosions (arrows), congestion and mild inflammation (asterisk) in the lamina propria one hour following ischemia in CUR group; (E) - intestinal morphology in the 
SHAM group six hours after surgery, diffuse interstitial inflammation of the lamina propria; Image (F) - transmural necrosis with complete loss of epithelia (arrows), severe edema and inflammatory infiltrate in the lamina propria, submucosa and muscularis six hours following ischemia; (G) - focal erosions, superficial congestion and inflammatory infiltrate of the lamina propria six hours following ischemia in RES group; $(\mathrm{H})$ - focal erosions (arrow) and inflammatory infiltrate (asterisk) of the lamina propria six hours following ischemia in CUR group. Scale bar was $100 \mu \mathrm{m}$. 
(a)

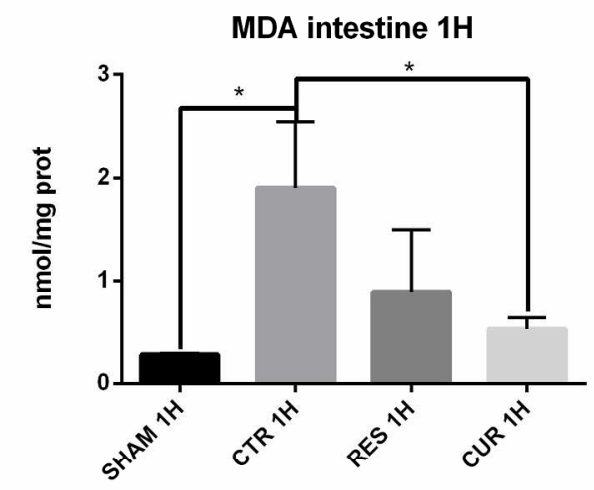

(c)

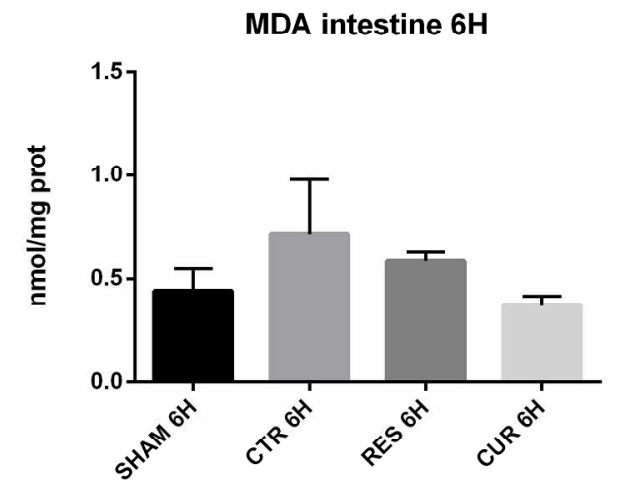

(b)

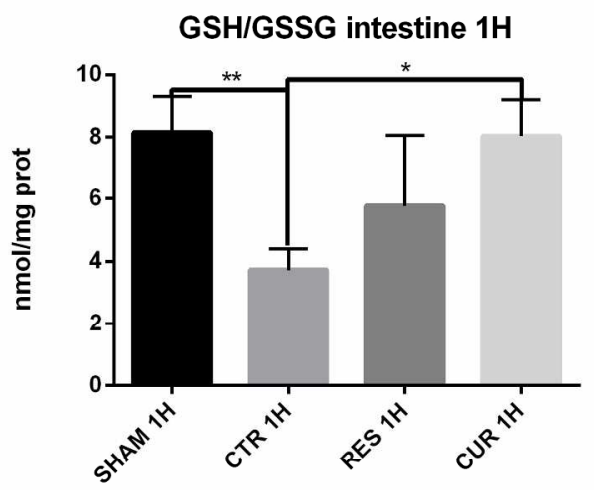

(d)

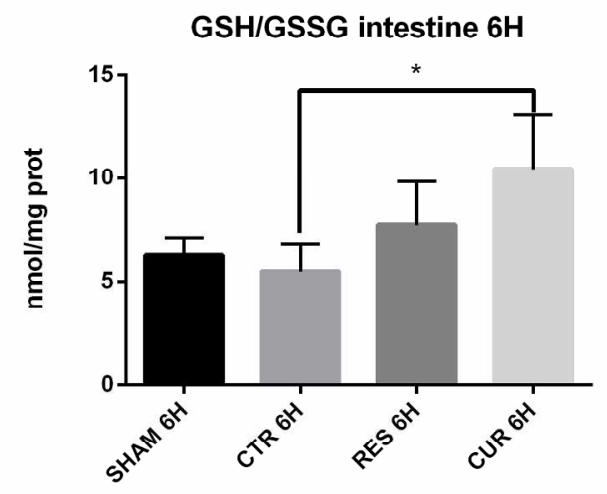

Figure 1- Oxidative stress parameters from serum in I/R after one week of pretreatment with RES and CUR.

(a) Malondialdehyde levels in serum after one hour of reperfusion; (b) GSH/GSSG ratio after one hour of reperfusion; (c) MDA production after six hours of reperfusion; (d) GSH/GSSG ratio after six hours of reperfusion. The statistical significance betwen compared groups was evaluated with one-way ANOVA followed by Bonferroni post-test, $* \mathrm{p}<0,5, * * \mathrm{p}<0,01, * * * \mathrm{p}<0,001, * * * * \mathrm{p}<0,0001$.

$$
201 \times 229 \mathrm{~mm}(300 \times 300 \mathrm{DPI})
$$


(a)

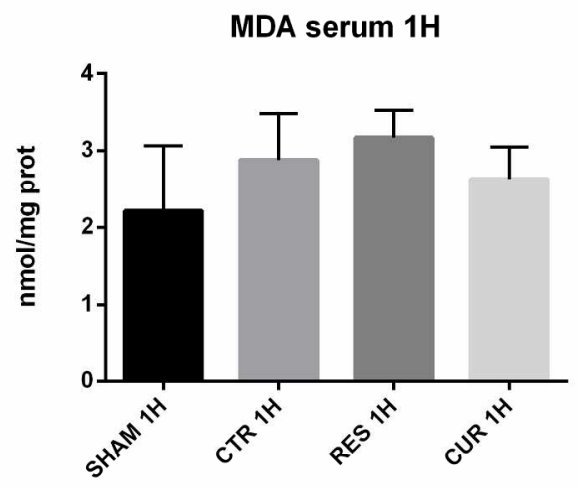

(c)

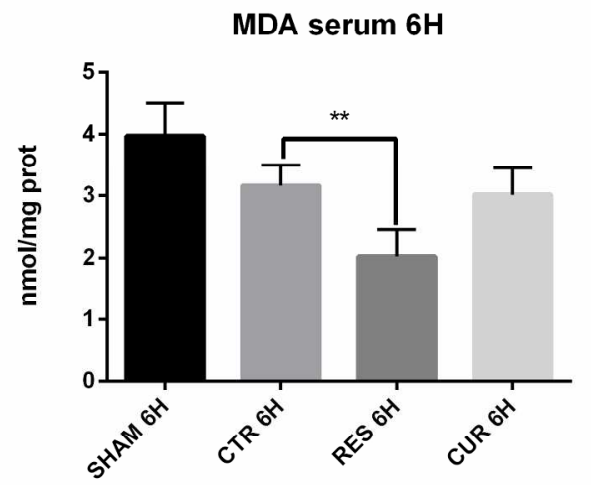

(b)

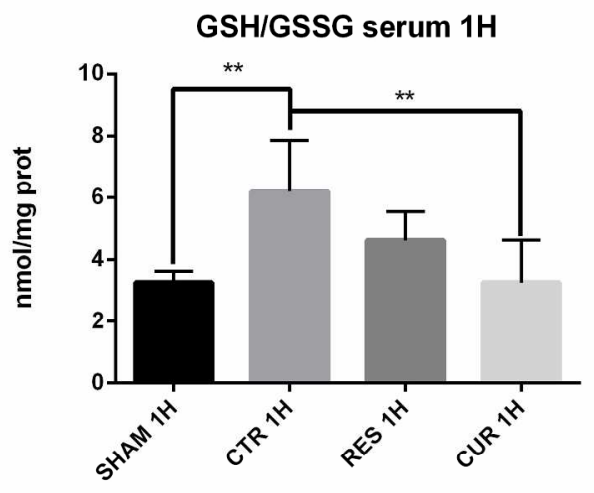

(d)

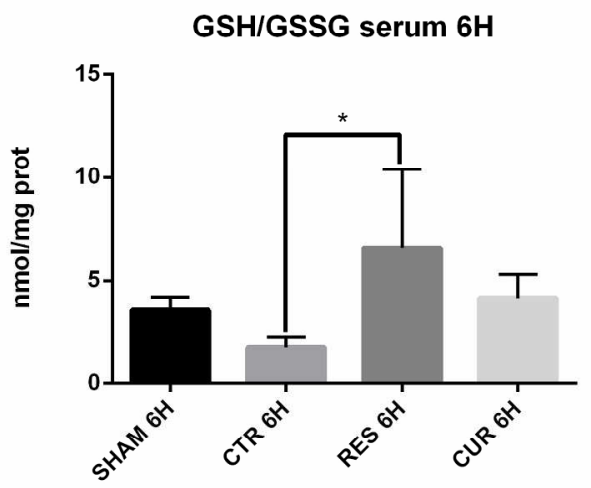

Figure 2 - Oxidative stress parameters in intestine samples in I/R after one week of pretreatment with RES and CUR. (a) Malondialdehyde levels after one hour of reperfusion; (b) GSH/GSSG ratio after one hour of reperfusion; (c) MDA formation after six hours of reperfusion; (d) GSH/GSSG ratio after six hours of reperfusion. The statistical significance betwen compared groups was evaluated with one-way ANOVA followed by Bonferroni post-test, $* p<0,5, * * p<0,01, * * * p<0,001, * * * * p<0,0001$. $202 \times 230 \mathrm{~mm}(300 \times 300$ DPI) 
(a)
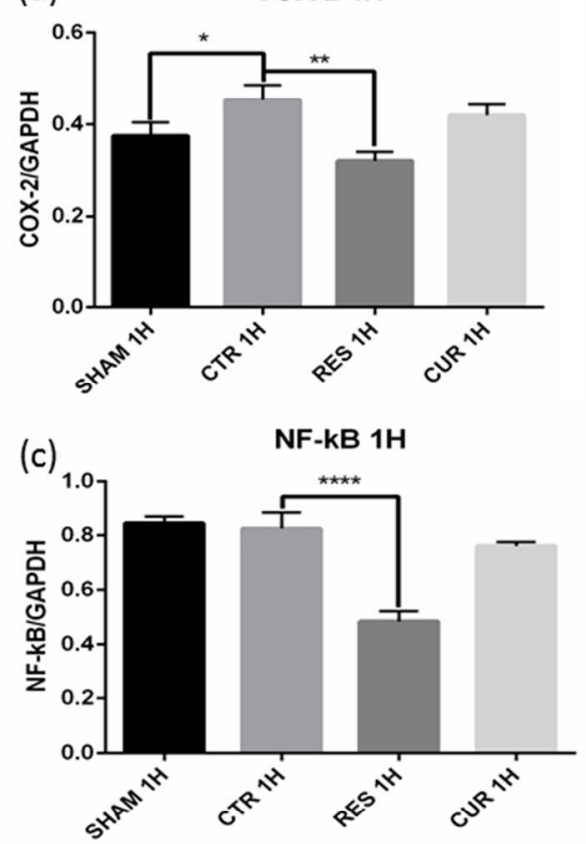

(e)

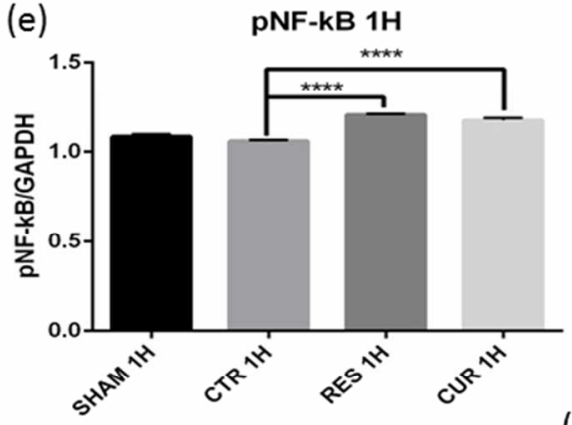

(b) $\quad$ cox-2 6H
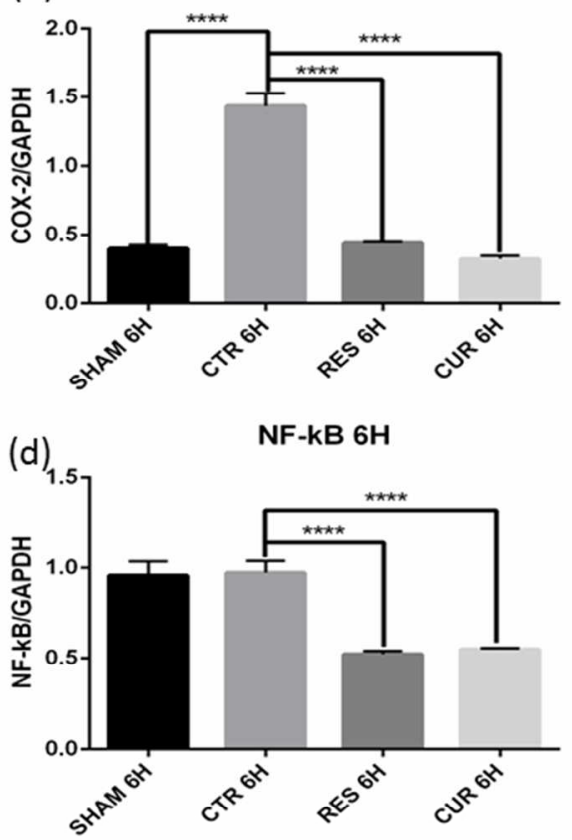

(f)

(g)
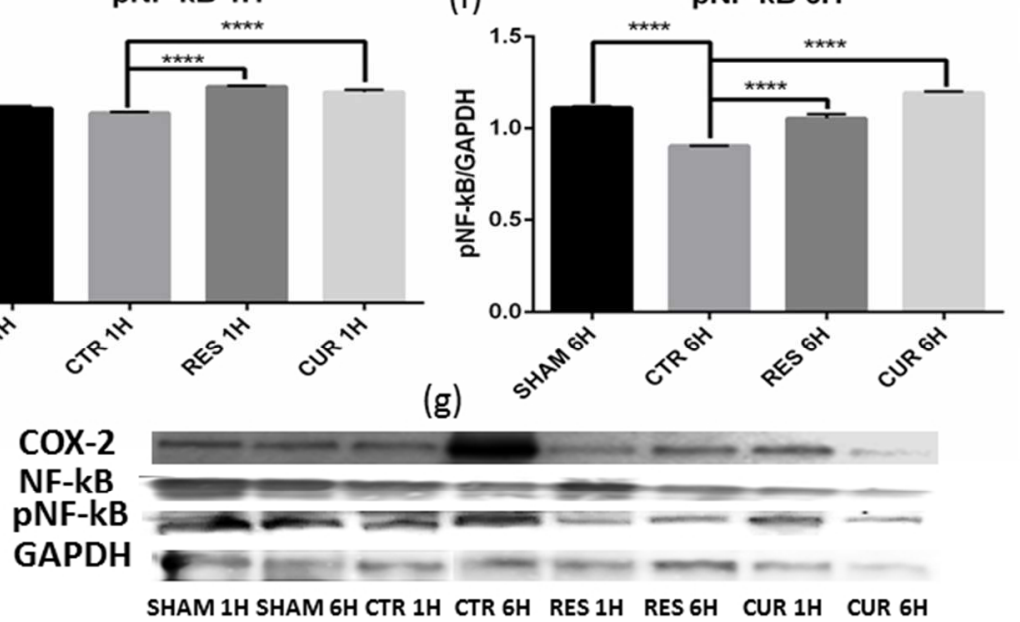

Figure 3 - Western Blot analysis of inflammatory markes in intestine samples in I/R after one week of pretreatment with RES and CUR. (a, b) COX-2 expressions after one hour and six hours of reperfusion.

Image analysis of Western blot bands' intensity was performed through densitometry and the data, normalized to GAPDH, are shown as graphs in upper panels. (c, d) NF-kB expressions after one hour and six hours of reperfusion; $(e, f)$ pNF-kB levels after one hour and six hours of reperfusion. (g) Representative images of immunoblotting for COX-2, NF-kB, pNF-kB and GAPDH in intestine homogenates are shown in lower panel. The statistical significance betwen compared groups was evaluated with one-way ANOVA followed by Bonferroni post-test, $* \mathrm{p}<0,5, * * \mathrm{p}<0,01, * * * \mathrm{p}<0,001, * * * * \mathrm{p}<0,0001$. $254 \times 338 \mathrm{~mm}(72 \times 72 \mathrm{DPI})$ 
(a)

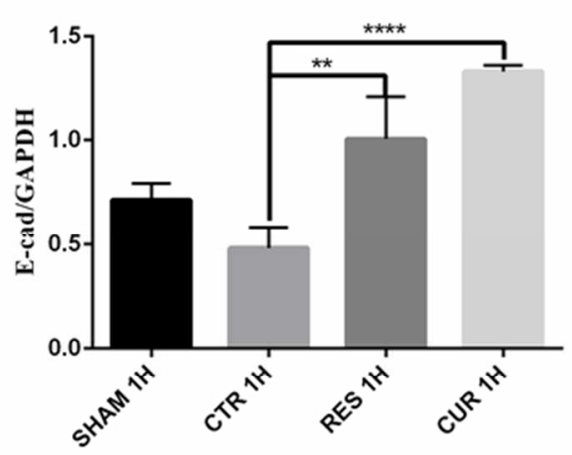

(c)

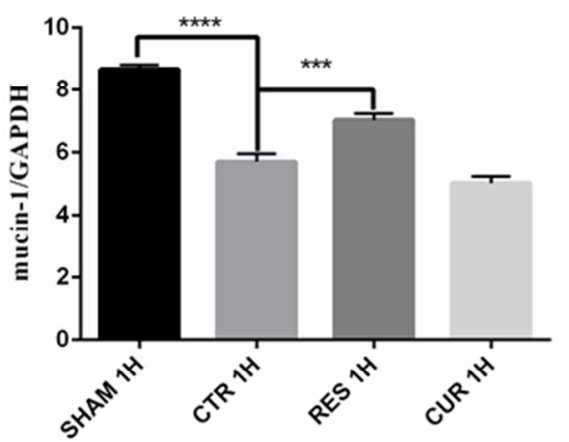

(b)

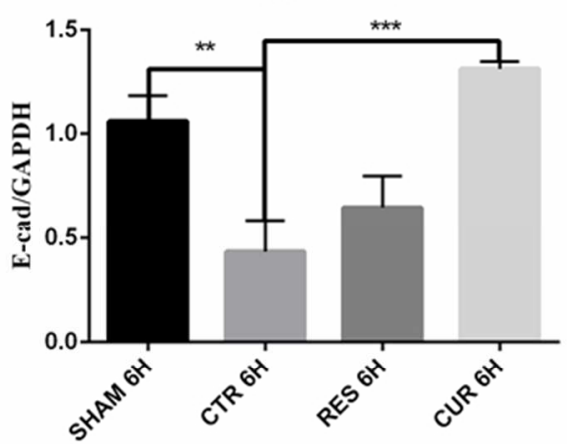

(d)

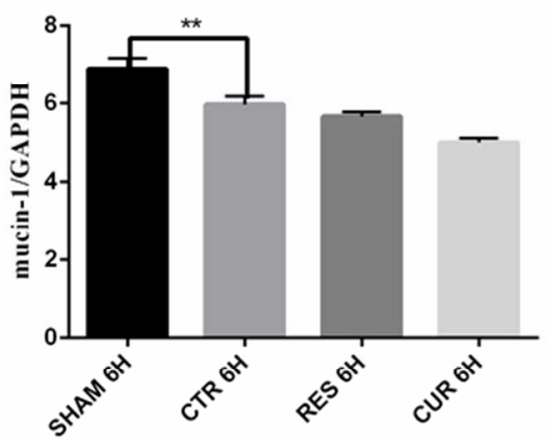

(e)

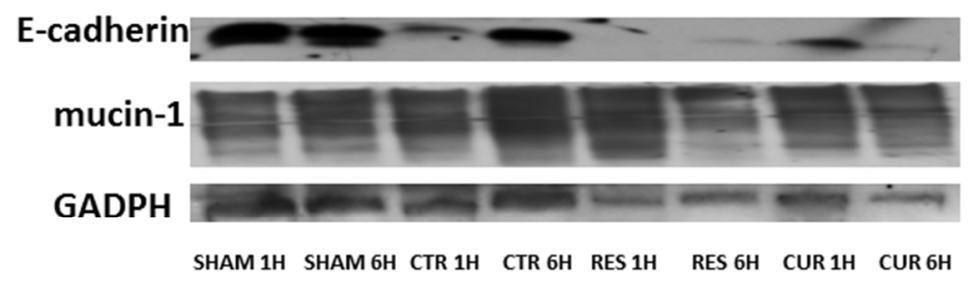

Figure 4 - E-cadherin and mucine-1 expressions in intestine samples in I/R after one week of pretreatment with RES and CUR. (a, b) E-cadherin expressions after one and six hour of reperfusion. Image analysis of Western blot bands' intensity was performed through densitometry and the data, normalized to GAPDH, are shown as graphs in upper panels.

(c, d) Mucin-1 levels after one hour and six hours of reperfusion. (e) Representative images of immunoblotting for E-cadherin, mucin-1 and GAPDH in intestine samples are shown in lower panel. The statistical significance betwen compared groups was evaluated with one-way ANOVA followed by Bonferroni post-test, $* p<0,5, * * p<0,01, * * * p<0,001, * * * * p<0,0001$. 
(a)

TRAIL 1H

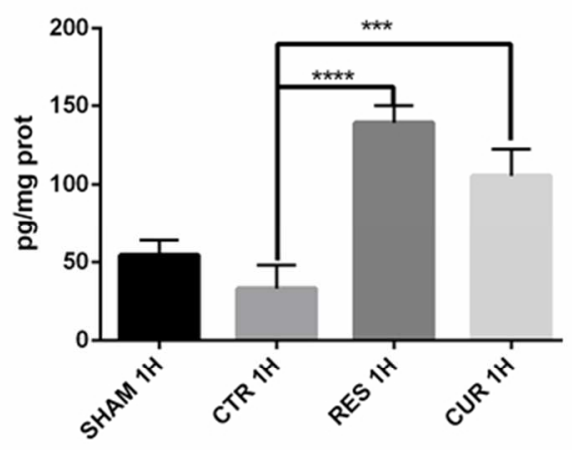

(b)

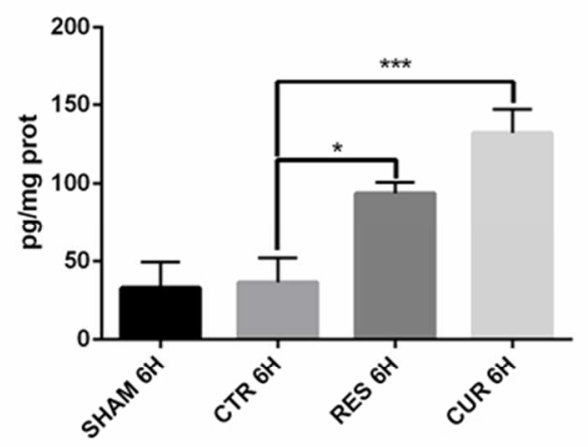

Figure 5 - TRAIL level in intestine samples in intestinal I/R after one week of pretreatment with RES and CUR. (a, b) TRAIL levels after one hour and six hours of reperfusion. The statistical significance betwen compared groups was evaluated with one-way ANOVA followed by Bonferroni post-test, $* p<0,5, * * p<0,01$, $* * * \mathrm{p}<0,001, * * * * \mathrm{p}<0,0001$.

$254 \times 338 \mathrm{~mm}(72 \times 72 \mathrm{DPI})$ 


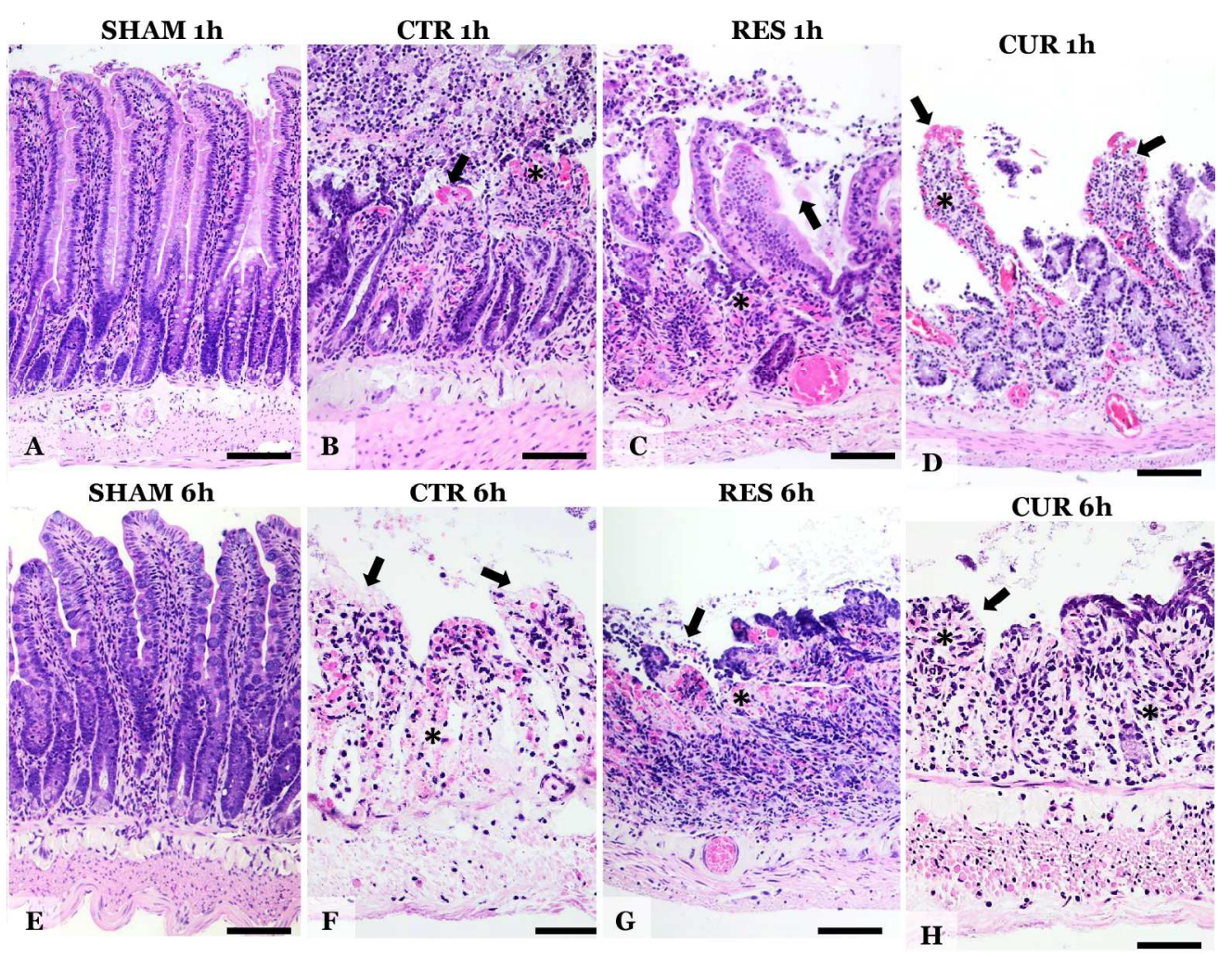

Figure 6 - Histopathological images in intestinal I/R after one week of pretreatment with RES and CUR. (A) normal intestinal morphology in the SHAM group one hour after surgery; $(B)$ - multifocal mucosal erosions and superficial necrosis (arrow) of the jejunum one hour following ischemia, severe congestion and mild inflammatory infiltrate (asterisk) of the lamina propria; (C) - focal mucosal erosions (arrows), congestion and mild inflammation (asterisk) in the lamina propria one hour following ischemia in RES pretreated group; (D) - diffuse intestinal erosions (arrows), congestion and mild inflammation (asterisk) in the lamina propria one hour following ischemia in CUR group; (E) - intestinal morphology in the SHAM group six hours after surgery, diffuse interstitial inflammation of the lamina propria; Image (F) - transmural necrosis with complete loss of epithelia (arrows), severe edema and inflammatory infiltrate in the lamina propria, submucosa and muscularis six hours following ischemia; (G) - focal erosions, superficial congestion and inflammatory infiltrate of the lamina propria six hours following ischemia in RES group; $(\mathrm{H})$ - focal erosions (arrow) and inflammatory infiltrate (asterisk) of the lamina propria six hours following ischemia in CUR group. Scale bar was $100 \mu \mathrm{m}$. $199 \times 153 \mathrm{~mm}(300 \times 300 \mathrm{DPI})$ 